\title{
ERRATUM
}

\author{
Z. P. Huang • J. Wang
}

\section{Erratum to: A theory of hyperelasticity of multi-phase media with surface/interface energy effect}

Published online: 15 October 2010

(c) Springer-Verlag 2010

\section{Erratum to: Acta Mechanica 182, 195-210 (2006) DOI 10.1007/s00707-005-0286-3}

We regret that in the expressions of the first kind Piola-Kirchhoff surface stress $\mathbf{S}_{s}$ and the Lagrangian description of the Young-Laplace equations (Eqs. 35-36), some terms associated with the "out-plane term" of surface gradient $\mathbf{F}_{s}$ and "out-plane term" of $\mathbf{S}_{s}$ were missing. In fact, $\mathbf{F}_{s}$ and $\mathbf{S}_{s}$ are "two-point" tensors, with base vectors both on the tangent planes $\mathcal{J}_{\mathrm{Y}}^{0}$ and $\mathcal{J}_{\mathrm{y}}$, and are not tensors in the 2-dimensional tangent plane $\mathcal{J}_{\mathrm{Y}}^{0}$ only. The correct forms of $\mathbf{F}_{s}, \mathbf{S}_{s}$ and the Lagrangian description of the Young-Laplace equations are given as follows.

(1) If the displacement on the surface $\mathbf{u}$ is decomposed into $\mathbf{u}_{0 s}=u_{0}^{\alpha} \mathbf{A}_{\alpha}$ in the tangent plane $\mathcal{J}_{\mathrm{Y}}^{0}$ and $\mathbf{u}_{0 n}=u_{0}^{n} \mathbf{A}_{3}$ along the normal direction of $\mathcal{J}_{\mathrm{Y}}^{0}$, then the surface gradient can be written as

$$
\mathbf{F}_{s}=\mathbf{a}_{\alpha} \otimes \mathbf{A}^{\alpha}=\mathbf{i}_{0}+\mathbf{u} \nabla_{0 s}+d_{0 \alpha} \mathbf{A}_{3} \otimes \mathbf{A}^{\alpha},
$$

where $\mathbf{i}_{0}$ is the second-order identity tensor on $\mathcal{J}_{\mathrm{Y}}^{0}, \mathbf{u} \nabla_{0 s}$ is defined as

$$
\mathbf{u} \nabla_{0 s}=\mathbf{u}_{0 s} \nabla_{0 s}-u_{0}^{n} \mathbf{b}_{0}=\left.u_{0}^{\lambda}\right|_{\alpha} \mathbf{A}_{\lambda} \otimes \mathbf{A}^{\alpha}-u_{0}^{n} \mathbf{b}_{0}
$$

and $d_{0 \alpha}=u_{0}^{\lambda} b_{0 \lambda \alpha}+\left.u_{0}^{n}\right|_{\alpha}, \mathbf{b}_{0}=b_{0 \lambda \alpha} \mathbf{A}^{\lambda} \otimes \mathbf{A}^{\alpha}$ is the curvature tensor of the surface in the reference configuration.

Equation (c1) can be expressed as the sum of an "in-plane term" ${ }^{\text {(in) }} \mathbf{F}_{s}=\mathbf{i}_{0}+\mathbf{u} \nabla_{0 s}$, and an "out-plane

term" ${ }^{\text {(ou })} \mathbf{F}_{s}=d_{0 \alpha} \mathbf{A}_{3} \otimes \mathbf{A}^{\alpha}$. Hence, the first kind Piola-Kirchhoff surface stress can also be decomposed into an "in-plane term" $\stackrel{(\text { in })}{\mathbf{S}_{s}}=\stackrel{(\text { in })}{\mathbf{F}_{s}} \cdot \stackrel{(1)}{\mathbf{T}_{s}}$, and an "out-plane term" $\stackrel{(\text { ou })}{\mathbf{S}_{s}}=\stackrel{(\text { ou })}{\mathbf{F}_{s}} \cdot \stackrel{(1)}{\mathbf{T}_{s}}$.

(2) $\delta \mathbf{u} \nabla_{0}$ and $\delta \mathbf{u} \nabla$ on the right-hand side of Eq. (21) (and hence on the left-hand side of Eqs. 22 and 23) should be replaced by $\delta \mathbf{a}_{\alpha} \otimes \mathbf{A}^{\alpha}=\delta \mathbf{F}_{s}$ and $\delta \mathbf{a}_{\alpha} \otimes \mathbf{a}^{\alpha}$, respectively.

Equation (22) should be

$$
\begin{aligned}
\mathbf{S}_{s}: \delta \mathbf{F}_{s}= & \stackrel{(\text { in })}{\mathbf{S}_{s}}: \delta \stackrel{(i n)}{\mathbf{F}_{s}}+\stackrel{(o u)}{\mathbf{S}_{s}}: \delta \stackrel{(o u)}{\mathbf{F}_{s}}=\left(\delta \mathbf{u}_{0 s} \cdot \stackrel{(i n)}{\mathbf{S}_{s}}\right) \cdot \nabla_{0 s}+\left(\delta u_{0}^{n} \mathbf{A}_{3} \cdot \stackrel{(o u)}{\mathbf{S}_{s}}\right) \cdot \nabla_{0 s} \\
& -\delta \mathbf{u}_{0 s} \cdot\left(\stackrel{(\text { in })}{\mathbf{S}_{s}} \cdot \nabla_{0 s}-\mathbf{b}_{0} \cdot \stackrel{(o u)^{\mathrm{T}}}{\mathbf{S}_{s}} \cdot \mathbf{A}_{3}\right)-\delta u_{0}^{n}\left(\stackrel{(i n)}{\mathbf{S}_{s}}: \mathbf{b}_{0}+\left(\mathbf{A}_{3} \cdot \stackrel{(o u)}{\mathbf{S}_{s}}\right) \cdot \nabla_{0 s}\right) .
\end{aligned}
$$

The online version of the original article can be found under doi:10.1007/s00707-005-0286-3.

Z. P. Huang $(\varangle) \cdot J$. Wang

LTCS and Department of Mechanics and Aerospace Engineering, College of Engineering,

Peking University, 100871 Beijing, China

E-mail: huangzp@pku.edu.cn 
$\sigma_{s}:(\delta \mathbf{u} \nabla)$ on the left hand side of Eqs. (23) and (27) should be $\sigma_{s}:\left(\delta \mathbf{a}_{\alpha} \otimes \mathbf{a}^{\alpha}\right)=\sigma_{s}:\left(\delta \mathbf{u} \nabla_{s}\right)$, where $\delta \mathbf{u} \nabla_{s}=\left.\delta u^{\alpha}\right|_{\beta} \mathbf{a}_{\alpha} \otimes \mathbf{a}^{\beta}-\delta u^{n} \mathbf{b}$.

Equation (26) should be

$$
\begin{aligned}
\int_{A_{0}} \mathbf{S}_{s}: \delta \mathbf{F}_{s} \mathrm{dA}_{0}= & -\int_{\partial \Omega_{0}} \delta \mathbf{u}_{0 s} \cdot \llbracket \stackrel{(i n)}{\mathbf{S}_{s}} \rrbracket \cdot \mathbf{n}_{0} \mathrm{~d} l_{0}-\int_{\partial \Omega_{0}} \delta u_{0}^{n} \mathbf{A}_{3} \cdot \llbracket \stackrel{(o u)}{\mathbf{S}_{s}} \rrbracket \cdot \mathbf{n}_{0} \mathrm{~d} l_{0} \\
& +\int_{A_{0}} \delta \mathbf{u}_{0 s} \cdot\left(-\stackrel{(i n)}{\mathbf{S}_{s}} \cdot \nabla_{0 s}+\mathbf{b}_{0} \cdot \stackrel{(o u)^{\mathrm{T}}}{\mathbf{S}_{s}} \cdot \mathbf{A}_{3}\right) \mathrm{dA}_{0} \\
& -\int_{A_{0}} \delta u_{0}^{n}\left(\stackrel{(i n)}{\mathbf{S}_{s}}: \mathbf{b}_{0}+\left(\mathbf{A}_{3} \cdot \stackrel{(o u)}{\mathbf{S}_{s}}\right) \cdot \nabla_{0 s}\right) \mathrm{dA}_{0} .
\end{aligned}
$$

Equations (35) and (36) should be written as

$$
\begin{aligned}
& \mathbf{A}_{3} \cdot \llbracket \mathbf{S}^{0} \rrbracket \cdot \mathbf{A}_{3}=-\left(\mathbf{S}_{s}^{(i n)}\right): \mathbf{b}_{0}-\left[\mathbf{A}_{3} \cdot\left(\mathbf{S}_{s}^{(o u)}\right)\right] \cdot \nabla_{0 s}, \\
& \mathbf{P}_{0} \cdot \llbracket\left[\mathbf{S}^{0} \rrbracket \cdot \mathbf{A}_{3}=-\left(\mathbf{S}_{s}^{(i n)}\right) \cdot \nabla_{0 s}+\left[\mathbf{A}_{3} \cdot\left(\mathbf{S}_{s}^{(o u)}\right) \cdot \mathbf{b}_{0}\right] .\right.
\end{aligned}
$$

It is noted that the basic idea, theoretical framework, and main results presented in the paper are not affected by these missing terms. 